\title{
Multi-Variable Model-Based Parameter Estimation Model for Antenna Radiation Pattern Prediction
}

\author{
Manohar D. Deshpande \& Robin L. Cravey, \\ NASA Langley Research Center, Hampton, VA. USA
}

\begin{abstract}
A new procedure is presented to develop multi-variable model-based parameter estimation (MBPE) model to predict far field intensity of antenna. By performing MBPE model development procedure on a single variable at a time, the present method requires solution of smaller size matrices. The utility of the present method is demonstrated by determining far field intensity due to a dipole antenna over a frequency range of $100-1000 \mathrm{MHz}$ and elevation angle range of $0-90$ degrees.
\end{abstract}

\section{Introduction}

Recently, there has been considerable interest in the use of the Model Based Parameter Estimation (MBPE) technique for improving the efficiency of computational methods used in electromagnetic analysis [1]. MBPE involves accurately fitting a mathematical model (Pade' rational function with unknown coefficients) to computed or measured quantities at a finite number of points. The unknown coefficients of the model are obtained from the solution of the resulting set of equations. Miller [2-3] describes in detail the theory behind MBPE and gives many examples to which MBPE may be applied. In most of the earlier work the Pade' rational function fitting model is used for MBPE in a single frequency variable. In [1], the Pade' rational function fitting model in the frequency domain is extended to include spatial dependence by making the numerator and denominator coefficients dependent on the spatial variable. The coefficients in the Pade' rational function are determined by solving a matrix equation with $N_{\theta} \times N_{f}$ unknowns, where $N_{\theta}$ and $N_{f}$ are, respectively, the number of sample points in the $\theta$ and $f$ dimensions. The technique described in [1], when extended to multi-parameter dependence, would require solution of a matrix equation with $N_{\theta} \times N_{f} \times N_{\phi} \times \ldots$. unknowns, where $N_{(\bullet)}$ is the number of sample points and $(\bullet)$ can be replaced by $\theta, f, \phi, \ldots \ldots$. In the present communication an alternative method to develop the MBPE method for two-parameter dependence is presented. Instead of requiring the solution of a matrix equation with $N_{\theta} \times N_{f}$ unknowns, as is done in [1], the present method requires solving a matrix equation with $N_{f}$ unknowns $N_{\theta}$ times to obtain the MBPE model for the frequency variable. The MBPE model for the $\theta$ variable is then obtained by solving a matrix equation with $N_{\theta}$ unknown $N_{f}$ times. One of the main advantages of this current approach is that the MBPE model for each variable is developed independently and hence requires smaller size matrix inversion.

\section{Theory}


Consider a dipole antenna of length $L$ and diameter $d_{0}$ placed along the $\mathrm{z}$ direction. The radiation pattern of the dipole as a function of frequency $f$ and elevation angle $\theta$ can be written as [1]

$$
|E|=F(f, \theta)=\frac{N_{0}(\theta)+N_{1}(\theta) f+N_{2}(\theta) f^{2}+. .+N_{n}(\theta) f^{n}}{1+D_{1}(\theta) f+D_{2}(\theta) f^{2}+. .+D_{d}(\theta) f^{d}}
$$

where $N_{0}, N_{1} \ldots \ldots N_{n}$ and $D_{1}, D_{d} \ldots . . D_{d}$ are unknown coefficients and depend only on $\theta$. The expression (1) is the required MBPE model with dependence on two parameters. The coefficients $N_{0}, N_{1} \ldots \ldots N_{n}$ and $D_{1}, D_{d} \ldots \ldots D_{d}$ can be expressed using

$$
A_{n}(\theta)=\frac{A_{n 0}+A_{n 1} \theta+A_{n 2} \theta^{2}+\ldots . . A_{n p} \theta^{p}}{1+A_{n(p+1)} \theta+A_{n(p+2)} \theta^{2}+\ldots . A_{n(p+q)} \theta^{q}}
$$

where $A_{n}(6)$ is either $N_{n}$ or $D_{n}$. The expression (2) is the MBPE models for the unknown coefficients appearing in (1). When $q=0$, the expressions (2) are identical to expression (7) in [1]. The unknown coefficients appearing in (2) can be calculated from a set of simultaneous equations formed by equating (1) to the calculated or measured electric field intensity due to the dipole at $(n+d+1)$ frequency points and, $(p+q+1)$ points in 6 space. If the procedure outlined in [1] is followed, the unknown coefficients are obtained by solving a matrix equation with $(p+q+1) *(n+d+1)$ number of unknowns. In this paper we present an alternative approach, which requires solving a matrix equation with $(n+d+1)$ or $(p+q+1)$ unknowns.

Consider the sampled electric field intensity due to the dipole for $\theta_{1}$ at $(n+d+1)$ frequency points and using $(1),(n+d+1)$ simultaneous equations are generated. The solution of these simultaneous equations are used to solve for $N_{0}, N_{1} \ldots \ldots N_{n}$, $D_{1}, D_{d} \ldots . . D_{d}$

Similarly, from the sampled data for $\theta_{2}, \theta_{3}, \ldots . . \theta_{(p+q+1)}$ at $(n+d+1)$ frequency points, coefficients $\left(N_{0}(), N_{1}() . . N_{n}(), D_{1}(), D_{2}() \ldots D_{d}()\right)$, at $(p+q+1) \quad \theta$ values are calculated. Now considering the coefficients $N_{0}\left(\theta_{1}\right), N_{0}\left(\theta_{2}\right), \ldots . N_{0}\left(\theta_{p+q+1)}\right)$ and using expression (2), $(p+q+1)$ simultaneous equations are generated and solved for $\left(A_{n 0}, A_{n 1} \ldots . A_{n p}, A_{n(p+1)}, A_{n(p+2)}, \ldots . A_{n(p+q)}\right)$. The electric field intensity at any $\theta$ and $f$ is obtained by first calculating the coefficients using expression (2), and then using expression (1).

\section{Numerical Results}

For numerical validation of the procedure described above, a dipole of length $L=0.4836$ meter and diameter $d_{0}=0.0003$ meter is considered. Using the Method of Moments (MoM), the far field intensity values due to the dipole at sample frequencies $f_{s}=0.15,0.30,0.45,0.60,0.75,0.90,0.95 \mathrm{GHz}$ and sample elevation angles $\epsilon_{s}=2 ., 10 ., 20 ., 40 ., 50 ., 60 ., 80 ., 90$. degrees are 
calculated. Selecting $(n=4, d=2)$ and $(p=5, q=2)$ various coefficients for MBPE are determined. The far field intensity for any $\square(0 \leq 6 \leq 90$ degrees $)$ over the frequency band $0 \leq f \leq 1.0 \mathrm{GHz}$ now is obtained from the knowledge of $\left(N_{00}, N_{01}, \ldots . N_{0(p+q)}\right),(n+d+1) \ldots$, etc., coefficients. Figures 1(a)-1(e) show far field intensities calculated at $\theta=10,30,50,70$ and 90 degrees over the frequency band using the MBPE model. Also plotted in Figures 1(a)-1(e) are the far field intensities calculated using the MoM. As can be seen from the figures, the results from the MBPE method presented in this paper are indistinguishable from the MOM calculations. In Figure 2, the far field intensity for this dipole as computed by the MBPE method presented in this paper is shown as a function of both frequency and theta.

\section{Conclusion}

A new method has been introduced in this communication to develop multi-variable MBPE model to predict an antenna radiation pattern as a function of frequency $f$ and spatial angle $\theta$. One of the main advantages of the new method is that the order of the matrix to be solved to determine MBPE parameters is much smaller than the earlier approach [1].

\section{References}

[1] Douglas H. Werner and Rene J. Allard, " The simultaneous interpolation of antenna radiation patterns in both the spatial and frequency domains using Model-Based Parameter Estimation," IEEE Trans. On Antennas and Propagation, Vol. 48, No. 3, pp. 383-392, March 2000.

[2] E. K. Miller, "Model-based parameter estimation in electromagnetics - I: Background and theoretical development," Applied Computational Electromagnetics Society Newsletter, Vol. 10, No. 3, pp. 40-63, 1995.

[3] -----,, “ Model based parameter estimation in electromagnetics---Part II: Application to EM observables," Applied Computational Electromagnetics Society Newsletter, Vol. 11, No. 1, pp.35-56, 1996.

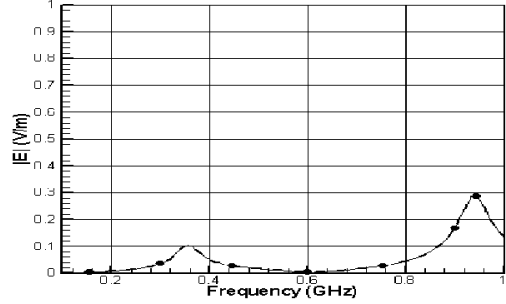

1.a. Theta $=10$ degrees

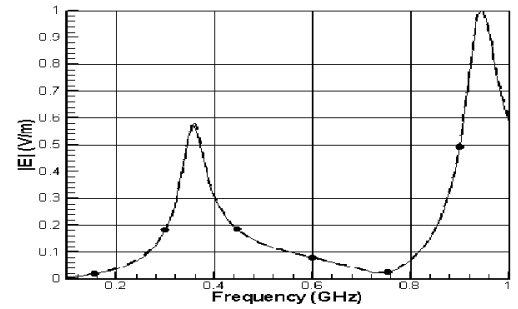

1.c. Theta $=50$ degrees

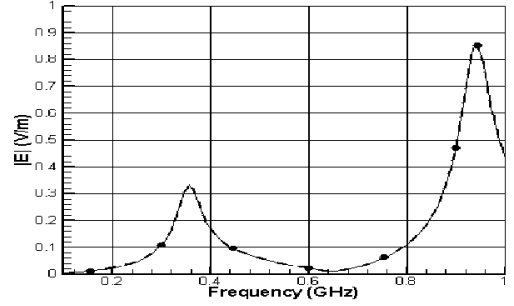

1.b. Theta $=30$ degrees

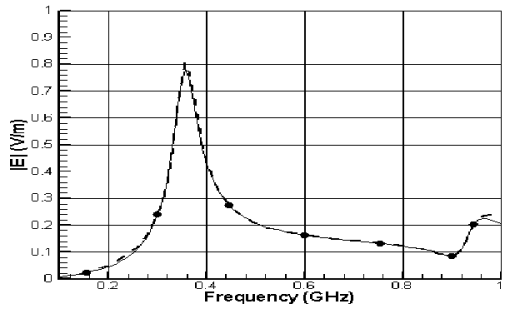

1.d. Theta $=70$ degrees 


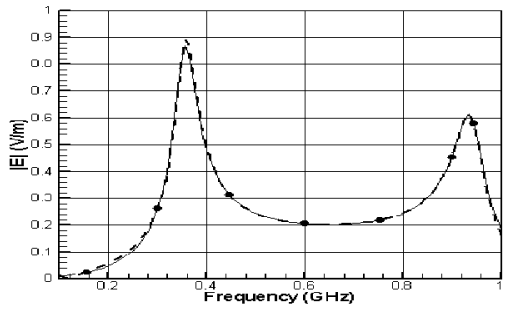

Method of moments

MBPE

Sample Points

1.e Theta $=90$ degrees

Figure 1. Far field intensity as a function of frequency for a dipole with length $L=0.4836$ meters and diameter $d_{0}=0.0003$ meters as calculated by MOM and MBPE at theta $=10,30,50,70$, and 90 degrees, with the seven fitting frequencies shown as points.

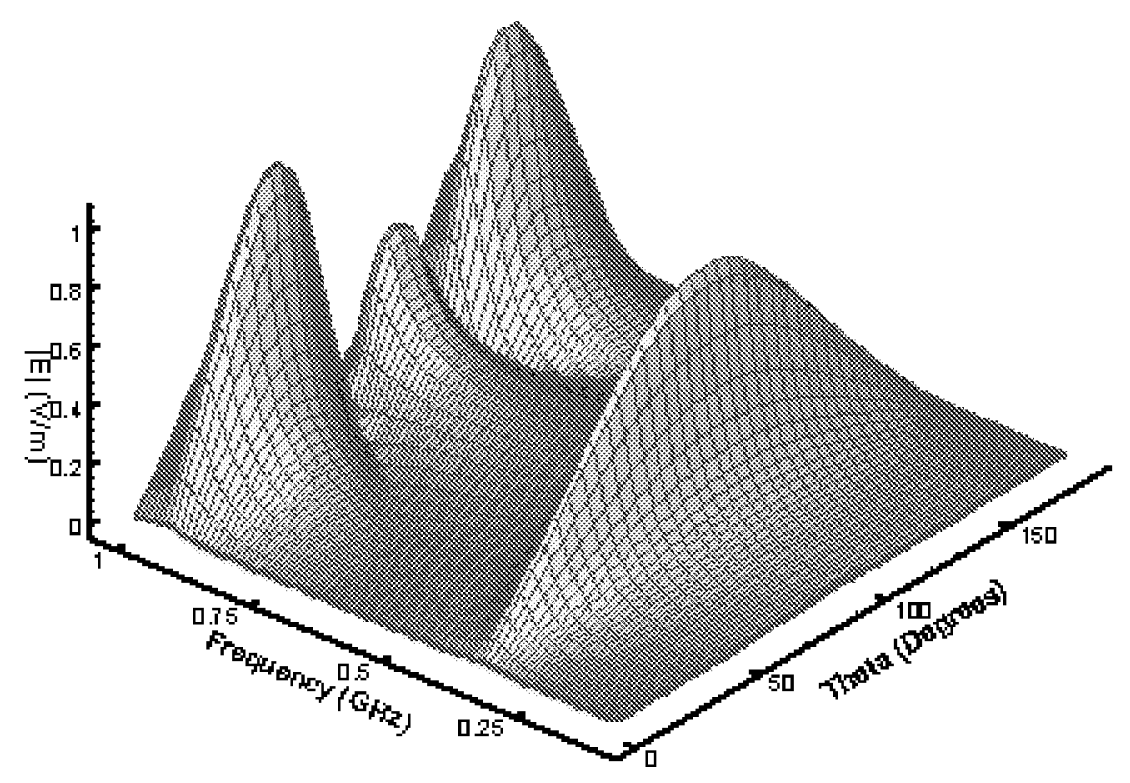

Figure 2. Far field intensity as a function of theta and frequency for a dipole of length $L=0.4836$ meters and diameter $d_{0}=0.0003$ meters 